\title{
EXISTENCE AND UNIQUENESS OF SOLUTION FOR A UNILATERAL PROBLEM IN SOBOLEV SPACES WITH VARIABLE EXPONENT
}

Abstract. We study the existence and uniqueness of the obstacle problem associated to the equation

$$
-\operatorname{div}(a(x, u, \nabla u)+\phi(u))+g(x, u)=f-\operatorname{div} F
$$

in the framework of Sobolev spaces with variable exponent, where $F \in$ $\left(L^{r(\cdot)}(\Omega)\right)^{N}$ and $f \in L^{q(\cdot)}(\Omega)$ with

$$
\begin{cases}r(x)>\frac{N}{p(x)-1}, \quad r(x) \geq p^{\prime}(x) & \forall x \in \Omega, \\ q(x)>\max \left(\frac{N}{p(x)}, 1\right), \quad q(x) \geq p^{\prime}(x) & \forall x \in \Omega,\end{cases}
$$

for a log-Lipschitz function $p: \bar{\Omega} \rightarrow[1,+\infty)$.

1. Introduction. Let $\Omega$ be a bounded open subset of $\mathbb{R}^{N}(N \geq 2)$, and $p(\cdot): \bar{\Omega} \rightarrow[1,+\infty)$ be a function satisfying the log-Lipschitz continuous condition such that $1<p_{-} \leq p_{+}<\infty$ (see Subsection 2.1).

The purpose of this paper is to study the obstacle and Dirichlet problem associated to the nonlinear elliptic equation

$$
-\operatorname{div}(a(x, u, \nabla u)+\phi(u))+g(x, u)=f-\operatorname{div}(F),
$$

2010 Mathematics Subject Classification: 35J87, 47J20, 35J66.

Key words and phrases: nonlinear obstacle problems, variable exponents, nonstandard growth, existence and uniqueness, $L^{\infty}$ estimate.

Received 24 May 2018; revised 1 August 2018 and 24 February 2019.

Published online 23 July 2019. 
when

$$
\begin{array}{ll}
f \in L^{q(\cdot)}(\Omega), & q(x)>\max \left(\frac{N}{p(x)}, 1\right), q(x) \geq p^{\prime}(x), \forall x \in \Omega, \\
F \in\left(L^{r(\cdot)}(\Omega)\right)^{N}, & r(x)>\frac{N}{p(x)-1}, r(x) \geq p^{\prime}(x), \forall x \in \Omega,
\end{array}
$$

and

- $a: \Omega \times \mathbb{R} \times \mathbb{R}^{N} \rightarrow \mathbb{R}^{N}$ is a Carathéodory function,

- $\phi \in C^{0}\left(\mathbb{R}, \mathbb{R}^{N}\right)$,

- $g$ is a Carathéodory function satisfying a sign condition.

The motivation for studying problem (1.1) comes from applications in elasticity [25] and non-Newtonian fluid mechanics [5, 21].

The solvability of (1.1) is very well understood in the case of $p$ constant (see [11, 12, 15, 17, 20]). When $p(\cdot)$ is a variable exponent, the existence of solutions to problem (1.1) has been obtained [23, 27] under some restrictive conditions on $F$ and $f$.

The novelty of this work is to refine and weaken the conditions on the data $F$ and $f$ and to show the existence and uniqueness of solution under conditions $(1.2)$ and $(1.3)$ on $f$ and $F$.

The main tool used is the result of Stampacchia [22] which yields the boundedness of solutions; inspired by the idea of [7], we partition $\bar{\Omega}$ into a finite number of balls $B_{i}$ such that for all continuous functions $f<g$ on $\Omega$, we have $\sup (f)<\inf (g)$ on each $B_{i}$, and for which the conditions of [22, Lemma 4] are satisfied.

This paper is organized as follows: in Section 2, we collect the necessary preliminaries and specify some assumptions; in Section 3, the existence of a bounded solution to problem (1.1) is established; and in the last section, the uniqueness of solution is proved.

\section{Preliminaries and assumptions}

2.1. Preliminaries. Let $\Omega$ be a bounded open subset of $\mathbb{R}^{N}(N \geq 2)$. We say that a real-valued continuous function $p(\cdot)$ is $\log$-Lipschitz continuous in $\Omega$ if

$$
-\log |x-y||p(x)-p(y)| \leq C \quad \forall x, y \in \bar{\Omega} \text { with } x \neq y \text { and }|x-y|<1 / 2,
$$

with a positive constant $C$. We denote

$C_{+}(\bar{\Omega})=\{\log$-Lipschitz continuous functions $p: \bar{\Omega} \rightarrow \mathbb{R}$ with

$$
\left.1<p_{-} \leq p_{+}<N\right\}
$$

where

$$
p_{-}=\min \{p(x): x \in \bar{\Omega}\}, \quad p_{+}=\max \{p(x): x \in \bar{\Omega}\} .
$$


For $p \in C_{+}(\bar{\Omega})$ we define the variable exponent Lebesgue space

$$
L^{p(\cdot)}(\Omega)=\left\{u: \Omega \rightarrow \mathbb{R} \text { measurable }: \int_{\Omega}|u(x)|^{p(x)} d x<\infty\right\} ;
$$

under the norm

$$
\|u\|_{p(\cdot)}=\inf \left\{\lambda>0: \int_{\Omega}\left|\frac{u(x)}{\lambda}\right|^{p(x)} d x \leq 1\right\},
$$

the space $L^{p(\cdot)}(\Omega)$ is a uniformly convex Banach space, and therefore reflexive. We denote by $L^{p^{\prime}(\cdot)}(\Omega)$ the conjugate space of $L^{p(\cdot)}(\Omega)$, where $1 / p(x)+$ $1 / p^{\prime}(x)=1$.

Proposition 2.1 (Generalized Hölder inequality [14, 24]).

(i) For any $u \in L^{p(\cdot)}(\Omega)$ and $v \in L^{p^{\prime}(\cdot)}(\Omega)$, we have

$$
\left|\int_{\Omega} u v d x\right| \leq\left(\frac{1}{p_{-}}+\frac{1}{p_{-}^{\prime}}\right)\|u\|_{p(\cdot)}\|v\|_{p^{\prime}(\cdot)} .
$$

(ii) For all $p_{1}, p_{2} \in C_{+}(\bar{\Omega})$ such that $p_{1}(x) \leq p_{2}(x)$ for all $x \in \Omega$, we have a continuous embedding

$$
L^{p_{2}(\cdot)}(\Omega) \hookrightarrow L^{p_{1}(\cdot)}(\Omega) .
$$

Proposition $2.2([14,24])$. Denote

$$
\rho(u)=\int_{\Omega}|u(x)|^{p(x)} d x \quad \forall u \in L^{p(\cdot)}(\Omega) .
$$

Then the following assertions hold:

(i) $\|u\|_{p(\cdot)}<1($ resp. $=1,>1)$ if and only if $\rho(u)<1($ resp. $=1,>1)$.

(ii) $\|u\|_{p(\cdot)}>1$ implies $\|u\|_{p(\cdot)}^{p_{-}} \leq \rho(u) \leq\|u\|_{p(\cdot)}^{p_{+}}$, while $\|u\|_{p(\cdot)}<1$ implies $\|u\|_{p(\cdot)}^{p_{+}} \leq \rho(u) \leq\|u\|_{p(\cdot)}^{p_{-}}$.

(iii) For a sequence $\left(u_{n}\right)_{n}$ in $L^{p(\cdot)}(\Omega),\left\|u_{n}\right\|_{p(\cdot)} \rightarrow 0$ if and only if $\rho\left(u_{n}\right) \rightarrow 0$, and $\left\|u_{n}\right\|_{p(\cdot)} \rightarrow \infty$ if and only if $\rho\left(u_{n}\right) \rightarrow \infty$.

Now, we define the variable exponent Sobolev space

$$
W^{1, p(\cdot)}(\Omega)=\left\{u \in L^{p(\cdot)}(\Omega):|\nabla u| \in L^{p(\cdot)}(\Omega)\right\},
$$

with the norm

$$
\|u\|_{1, p(\cdot)}=\|u\|_{p(\cdot)}+\|\nabla u\|_{p(\cdot)} \quad \forall u \in W^{1, p(\cdot)}(\Omega) .
$$

We denote by $W_{0}^{1, p(\cdot)}(\Omega)$ the closure of $C_{0}^{\infty}(\Omega)$ in $W^{1, p(\cdot)}(\Omega)$, and we define the Sobolev exponent by

$$
p^{*}(x)=\frac{N p(x)}{N-p(x)} \quad \text { for } p(x)<N .
$$


Proposition 2.3 ([14]).

(i) If $1<p_{-} \leq p_{+}<\infty$, then the spaces $W^{1, p(\cdot)}(\Omega)$ and $W_{0}^{1, p(\cdot)}(\Omega)$ are separable and reflexive Banach spaces.

(ii) If $q \in C_{+}(\bar{\Omega})$ and $q(x)<p^{*}(x)$ for any $x \in \Omega$, then the embedding $W_{0}^{1, p(\cdot)}(\Omega) \hookrightarrow \hookrightarrow L^{q(\cdot)}(\Omega)$ is continuous and compact.

(iii) Poincaré inequality: there exists a constant $C>0$ such that

$$
\|u\|_{p(\cdot)} \leq C\|\nabla u\|_{p(\cdot)} \quad \forall u \in W_{0}^{1, p(\cdot)}(\Omega) .
$$

(vi) Sobolev-Poincaré inequality: there exists another constant $C>0$ such that

$$
\|u\|_{p *(\cdot)} \leq C\|\nabla u\|_{p(\cdot)} \quad \forall u \in W_{0}^{1, p(\cdot)}(\Omega) .
$$

Remark 1. By Proposition 2.3(iii), the norms $\|\nabla u\|_{p(\cdot)}$ and $\|u\|_{1, p(\cdot)}$ are equivalent in $W_{0}^{1, p(\cdot)}(\Omega)$.

LEMma 2.4 ([6]). Let $F: \mathbb{R} \rightarrow \mathbb{R}$ be a uniformly Lipschitz function with $F(0)=0$ and let $p \in C_{+}(\bar{\Omega})$. If $u \in W_{0}^{1, p(\cdot)}(\Omega)$, then $F(u) \in W_{0}^{1, p(\cdot)}(\Omega)$. Moreover, if the set $D$ of discontinuity points of $F^{\prime}$ is finite, then

$$
\frac{\partial(F \circ u)}{\partial x_{i}}= \begin{cases}F^{\prime}(u) \frac{\partial u}{\partial x_{i}} & \text { a.e. in }\{x \in \Omega: u(x) \notin D\}, \\ 0 & \text { a.e. in }\{x \in \Omega: u(x) \in D\} .\end{cases}
$$

LEMMA 2.5 (6]). Under assumptions $\left(H_{1}\right)-\left(H_{6}\right)$ below, let $\left(u_{n}\right)_{n}$ be a sequence in $W_{0}^{1, p(\cdot)}(\Omega)$ such that $u_{n} \rightarrow u$ in $W_{0}^{1, p(\cdot)}(\Omega)$ and

$$
\int_{\Omega}\left[a\left(x, u_{n}, \nabla u_{n}\right)-a\left(x, u_{n}, \nabla u\right)\right] \nabla\left(u_{n}-u\right) \rightarrow 0 .
$$

Then $u_{n} \rightarrow u$ in $W_{0}^{1, p(\cdot)}(\Omega)$.

2.2. Assumptions. Let

$\left(H_{1}\right) a: \Omega \times \mathbb{R} \times \mathbb{R}^{N} \rightarrow \mathbb{R}^{N}$ is a Carathéodory function such that for some $\alpha>0$,

$$
a(x, s, \xi) \cdot \xi \geq \alpha|\xi|^{p(x)}, \quad \forall s \in \mathbb{R}, \text { a.e. } x \in \Omega, \forall \xi \in \mathbb{R}^{N} .
$$

$\left(H_{2}\right)(1)\left[a(x, s, \xi)-a\left(x, s, \xi^{\prime}\right)\right]\left[\xi-\xi^{\prime}\right]>0$ for a.e. $x \in \Omega$, all $s \in \mathbb{R}$, and all $\xi, \xi^{\prime} \in \mathbb{R}^{N}$ with $\xi \neq \xi^{\prime}$,

(2) there is an increasing function $\beta: \mathbb{R}^{+} \rightarrow \mathbb{R}^{+}$and a non-negative function $\bar{\beta} \in L^{p^{\prime}(\cdot)}(\Omega)$ with $|a(x, s, \xi)| \leq \beta(|s|)\left[|\xi|^{p(x)-1}+\bar{\beta}(x)\right]$ for a.e. $x \in \Omega$, all $s \in \mathbb{R}$ and all $\xi \in \mathbb{R}^{N}$.

$\left(H_{3}\right) f \in L^{q(\cdot)}(\Omega), F \in\left(L^{r(\cdot)}(\Omega)\right)^{N}$, where $q(x) \geq 1$ and $r(x) \geq p^{\prime}(x)$ for all $x \in \Omega$.

$\left(H_{4}\right) g: \Omega \times \mathbb{R} \rightarrow \mathbb{R}$ is a Carathéodory function such that $\sup _{|s| \leq n}|g(\cdot, s)|=$ $h_{n}(\cdot) \in L^{1}(\Omega)$ and $g(x, s) s \geq 0$ for a.e. $x \in \Omega$ and all $s \in \mathbb{R}$. 
$\left(H_{5}\right) \phi: \mathbb{R} \rightarrow \mathbb{R}^{N}$ is continuous.

$\left(H_{6}\right) \psi \in L^{\infty}(\Omega)$ and $K(\psi)=\left\{v \in W_{0}^{1, p(\cdot)}(\Omega): v \geq \psi\right.$ a.e. in $\left.\Omega\right\} \neq \emptyset$.

Definition 2.6. For all $k>0$ and $s \in \mathbb{R}$, the truncation function $T_{k}(\cdot)$ is defined by

$$
T_{k}(s)= \begin{cases}s & \text { if }|s| \leq k, \\ k \cdot \operatorname{sign}(s) & \text { if }|s|>k,\end{cases}
$$

and we set

$$
G_{k}(s)=s-T_{k}(s) .
$$

Definition 2.7. A measurable function $u \in K(\psi)$ is called a weak solution of the unilateral problem $\sqrt{1.1}$ if $a(x, u, \nabla u) \in\left(L^{p^{\prime}(\cdot)}(\Omega)\right)^{N}$ and $g(x, u) \in L^{1}(\Omega)$, and for all $v \in K(\psi) \cap L^{\infty}(\Omega)$,

$$
\begin{array}{r}
\int_{\Omega} a(x, u, \nabla u) \nabla(u-v) d x+\int_{\Omega} \phi(u) \nabla(u-v) d x+\int_{\Omega} g(x, u)(u-v) d x \\
\leq \int_{\Omega} f(u-v) d x+\int_{\Omega} F \nabla(u-v) d x .
\end{array}
$$

THEOREM 2.8. Suppose that assumptions $\left(H_{1}\right)-\left(H_{6}\right)$ hold, and let $q(x)>$ $\max \left(\frac{N}{p(x)}, p^{\prime}(x)\right)$ and $r(x)>\frac{N}{p(x)-1}$. Then any weak solution $u$ to problem (1.1) (in the sense of definition (2.7)) is bounded.

Proof. For fixed $k, h, \theta>0$, define $\omega_{n}=\frac{1}{h} T_{h}\left(G_{k}\left(T_{n}(u)\right)\right.$ and $\bar{\omega}_{n}=\theta \omega_{n}$, where $k=\theta+\|\psi\|_{L^{\infty}(\Omega)}$.

Note that $v=T_{n}(u)-\bar{\omega}_{n} \in K(\psi) \cap L^{\infty}(\Omega)$. Taking $v$ as a test function in (2.1), we obtain

$$
\begin{aligned}
\int_{\Omega} a(x, u, & \nabla u) \cdot \nabla\left(\bar{\omega}_{n}+u-T_{n}(u)\right) d x+\int_{\Omega} \phi(u) \cdot \nabla\left(\bar{\omega}_{n}+u-T_{n}(u)\right) \\
& \quad+\int_{\Omega} g(x, u)\left(\bar{\omega}_{n}+u-T_{n}(u)\right) \\
\leq & \int_{\Omega} f\left(\bar{\omega}_{n}+u-T_{n}(u)\right) d x+\int_{\Omega} F \nabla\left(\bar{\omega}_{n}+u-T_{n}(u)\right) d x .
\end{aligned}
$$

Setting $\bar{\omega}=\frac{\theta}{h} T_{h}\left(u-G_{k}(u)\right)$, we have

$$
\bar{\omega}_{n} \rightarrow \bar{\omega} \quad \text { strongly in } W_{0}^{1 . p(\cdot)}(\Omega)
$$

and

$$
\nabla \bar{\omega}=\frac{\theta}{h} \nabla \chi_{\{k \leq|u| \leq k+h\}} \quad \text { a.e. in } \Omega .
$$


Passing to the limit in 2.2 , we get

$$
\begin{aligned}
\frac{1}{h} \int_{\{k<|u|<k+h\}} a(x, u, \nabla u) \cdot \nabla u d x+\frac{1}{h} \int_{\{k<|u|<k+h\}} \phi(u) \cdot \nabla u d x \\
\quad+\frac{1}{h} \int_{\Omega} g(x, u) T_{h}\left(u-G_{k}(u)\right) d x \\
\leq \frac{1}{h} \int_{\Omega} f(x) T_{h}\left(u-G_{k}(u)\right) d x+\frac{1}{h} \int_{\Omega} g(x, u) T_{h}\left(u-G_{k}(u)\right) d x \\
\quad+\int_{\{k<|u|<k+h\}} F \cdot \nabla u d x .
\end{aligned}
$$

By $\left(H_{5}\right)$, we may assume that $\phi=\left(\phi_{1}, \ldots, \phi_{N}\right)$, where $\phi_{i} \in C(\mathbb{R})$ for $1 \leq i \leq N$.

Let $\tilde{\phi}_{i}(t)=\int_{0}^{t} \chi_{\{k<|\eta|<k+h\}} \phi_{i}(\eta) d \eta$ and set $\tilde{\phi}=\left(\tilde{\phi}_{1}, \ldots, \tilde{\phi}_{N}\right)$. Then it is easy to see that $\tilde{\phi} \in\left(W_{0}^{1, p(\cdot)}(\Omega)\right)^{N}$. Thus, for the second term of the left-hand side in $(2.3)$, using Lemma 2.4 , we have

$$
\begin{aligned}
\int_{\{k<|u|<k+h\}} \phi(u) \cdot \nabla u d x & =\int_{\Omega} \chi_{\{k<|u|<k+h\}} \phi(u) \cdot \nabla u d x \\
& =\int_{\Omega} \operatorname{div} \tilde{\phi}(u) d x=0 .
\end{aligned}
$$

Combining (2.3) with (2.4), it follows from $\left(H_{1}\right)$ and $\left(H_{4}\right)$ that

$$
\alpha \int_{\{k<|u|<k+h\}}|\nabla u|^{p(x)} d x \leq \int_{\{|u|>k\}}|f(x)|\left|G_{k}(u)\right| d x+\int_{\{k<|u|<k+h\}}|F||\nabla u| d x .
$$

Letting $h$ tend to infinity in $(2.5)$, we obtain

$$
\alpha \int_{A(k)}|\nabla u|^{p(x)} d x \leq \int_{A(k)}|f(x)|\left|G_{k}(u)\right| d x+\int_{A(k)}|F||\nabla u| d x,
$$

where $A(k)=\{x \in \Omega:|u(x)|>k\}$.

By using the Young inequality in the second term on the right hand side of (2.6) we have

$$
\int_{\Omega} F \cdot \nabla G_{k}(u) d x \leq c_{1} \int_{A(k)}|F|^{p^{\prime}(x)} d x+c_{2} \int_{\Omega}\left|\nabla G_{k}(u)\right|^{p(x)} d x .
$$

Now combining the last two formulas, using Proposition 2.2, and taking 


$$
\begin{aligned}
& c^{\prime}=\alpha-c_{2}>0 \text {, we get } \\
& \qquad \begin{array}{l}
c_{\Omega}^{\prime}\left|\nabla G_{k}(u)\right|^{p(x)} d x \leq c_{1} \int_{A(k)}|F|^{p^{\prime}(x)} d x+\int_{\Omega}|f| \cdot\left|G_{k}(u)\right| d x \\
\quad \leq c_{1} \int_{A(k)}|F|^{p^{\prime}(x)} d x+c_{3}\left\|f \chi_{A_{k}}\right\|_{p_{*}^{\prime}(\cdot)} \cdot\left\|G_{k}(u)\right\|_{p_{*}(\cdot)} \\
\quad \leq c_{1} \int_{A(k)}|F|^{p^{\prime}(x)} d x+c_{3}\left\|f \chi_{A_{k}}\right\|_{p_{*}^{\prime}(\cdot)} \cdot\left\|\nabla G_{k}(u)\right\|_{p(\cdot)} \\
\quad \leq c_{1} \int_{A(k)}|F|^{p^{\prime}(x)} d x+c_{3}\left\|f \chi_{A_{k}}\right\|_{p_{*}^{\prime}(\cdot)}\left(\int_{\Omega}\left|\nabla G_{k}(u)\right|^{p(x)} d x\right)^{1 / \gamma_{1}}
\end{array}
\end{aligned}
$$

with

$$
\gamma_{1}= \begin{cases}p^{-} & \text {if }\left\|\nabla G_{k}(u)\right\|_{p(\cdot)} \geq 1 \\ p^{+} & \text {if }\left\|\nabla G_{k}(u)\right\|_{p(\cdot)}<1 .\end{cases}
$$

Using Young's inequality, we obtain

$$
\begin{gathered}
c_{\Omega} \int_{\Omega}\left|\nabla G_{k}(u)\right|^{p(x)} d x \leq c_{1} \int_{A(k)}|F|^{p^{\prime}(x)} d x+c_{1}^{\prime}\left\|f \chi_{A_{k}}\right\|_{p_{*}^{\prime}(\cdot)}^{\gamma_{1}^{\prime}} \\
+\frac{c^{\prime}}{2} \int_{\Omega}\left|\nabla G_{k}(u)\right|^{p(x)} d x .
\end{gathered}
$$

By Hölder's inequality and Proposition 2.2, we get

$$
\begin{aligned}
& \frac{c^{\prime}}{2} \int_{\Omega}\left|\nabla G_{k}(u)\right|^{p(x)} d x \leq c_{1} \int_{A(k)}|F|^{p^{\prime}(x)} d x+c_{1}^{\prime}\left(\int_{A(k)}|f|^{p_{*}^{\prime}(x)}\right)^{\gamma_{1}^{\prime} / \gamma_{2}} d x \\
& \leq c_{1} \int_{A(k)}|F|^{p^{\prime}(x)} d x+c_{1}^{\prime}\left\||f|^{p_{*}^{\prime}}\right\|_{L^{s_{2}(\cdot) / p_{*}^{\prime}(\cdot)}(\Omega)}^{\gamma_{1}^{\prime} / \gamma_{2}} \cdot\left\|\chi_{A_{k}}\right\|_{L^{\frac{s_{2}(\cdot)-p_{*}^{\prime}(\cdot)}{s_{2}(\Omega)}}}^{\gamma_{1}^{\prime} / \gamma_{2}} \\
& \leq c_{1} \int_{A(k)}|F|^{p^{\prime}(x)} d x+c_{3}^{\prime}(\Phi(k))^{\frac{\gamma_{1}^{\prime}}{\gamma_{2} \cdot \gamma_{5}}} \\
& \leq c_{1}\left\||F|^{p^{\prime}(x)}\right\|_{s_{1}(\cdot) / p^{\prime}(\cdot)} \cdot(\Phi(k))^{\frac{1}{\gamma_{6}}}+c_{3}^{\prime}(\Phi(k))^{\frac{\gamma_{1}^{\prime}}{\gamma_{2} \cdot \gamma_{5}}}
\end{aligned}
$$

with $s_{1}(x)>r(x)$ and $s_{2}(x)>q(x)$ for $x \in \Omega, \Phi(k)=\operatorname{meas}(A(k))$, and

$$
\begin{aligned}
& \gamma_{2}= \begin{cases}p_{*}^{\prime-} & \text { if }\left\|f \chi_{A_{k}}\right\|_{p_{*}^{\prime}(\cdot)} \geq 1, \\
p_{*}^{\prime+} & \text { if }\left\|f \chi_{A_{k}}\right\|_{p_{*}^{\prime}(\cdot)}<1,\end{cases} \\
& \gamma_{5}= \begin{cases}\left(\frac{s_{2}(x)}{s_{2}(x)-p_{*}^{\prime}(x)}\right)^{-} & \text {if }\left\|\chi_{A_{k}}\right\|_{\frac{s_{2}(\cdot)}{s_{2}(\cdot)-p_{*}^{\prime}(\cdot)} \geq 1,} \\
\left(\frac{s_{2}(x)}{s_{2}(x)-p_{*}^{\prime}(x)}\right)^{+} & \text {if }\left\|\chi_{A_{k}}\right\|_{\frac{s_{2}(\cdot)}{s_{2}(\cdot)-p_{*}^{\prime}(\cdot)}}<1,\end{cases}
\end{aligned}
$$




$$
\gamma_{6}= \begin{cases}\left(\frac{s_{1}(x)}{s_{1}(x)-p^{\prime}(x)}\right)^{-} & \text {if }\left\|\chi_{A_{k}}\right\|_{\frac{s_{1}(\cdot)}{s_{1}(\cdot)-p^{\prime}(\cdot)} \geq 1} \\ \left(\frac{s_{1}(x)}{s_{1}(x)-p^{\prime}(x)}\right)^{+} & \text {if }\left\|\chi_{A_{k}}\right\|_{\frac{s_{1}(\cdot)}{s_{1}(\cdot)-p^{\prime}(\cdot)}}<1 .\end{cases}
$$

In view of the Sobolev inequality and Proposition 2.2, we have

$$
\int_{\Omega}\left|\nabla G_{k}(u)\right|^{p(x)} d x \geq c_{4}\left(\int_{\Omega}\left|G_{k}(u)\right|^{p_{*}(x)}\right)^{\gamma_{4} / \gamma_{3}} d x,
$$

where

$$
\gamma_{3}=\left\{\begin{array}{ll}
\left(p_{*}\right)^{-} & \text {if }\left\|G_{k}(u)\right\|_{p_{*}(\cdot)} \geq 1, \\
\left(p_{*}\right)^{+} & \text {if }\left\|G_{k}(u)\right\|_{p_{*}(\cdot)}<1 .
\end{array} \quad \gamma_{4}= \begin{cases}p^{-} & \text {if }\left\|\nabla G_{k}(u)\right\|_{p(\cdot)} \geq 1, \\
p^{+} & \text {if }\left\|\nabla G_{k}(u)\right\|_{p(\cdot)}<1 .\end{cases}\right.
$$

So by (2.7) and (2.8), we obtain

$$
\int_{\Omega}\left|G_{k}(u)\right|^{p_{*}(x)} d x \leq c \max \left((\Phi(k))^{\frac{\gamma_{1}^{\prime} \cdot \gamma_{3}}{\gamma_{2} \cdot \gamma_{5} \cdot \gamma_{4}}} ;(\Phi(k))^{\frac{\gamma_{3}}{\gamma_{6} \cdot \gamma_{4}}}\right) .
$$

Choose $h$ such that $h-k>1$ and in $A_{h}=\{x \in \Omega:|u|>h\}$ we have $h-k<G_{k}(u)$. Then in view of 2.9 we get

$$
\Phi(h) \leq \frac{C}{(h-k)^{p_{*}^{-}}} \max \left((\Phi(k))^{\frac{\gamma_{1}^{\prime} \cdot \gamma_{3}}{\gamma_{2} \cdot \gamma_{5} \cdot \gamma_{4}}} ;(\Phi(k))^{\frac{\gamma_{3}}{\gamma_{6} \cdot \gamma_{4}}}\right) .
$$

First, let $p^{+}$be a constant satisfying $p^{+}<\min _{x \in \bar{\Omega}}(1+1 / N) p(x)$, which implies that

$$
p^{+}<\min _{x \in \bar{\Omega}} \frac{N p(x)}{N-p(x)} .
$$

Then $\gamma_{3} / \gamma_{4}>1$ and $\gamma_{1}^{\prime} / \gamma_{2}>1$. By a suitable choice of $s_{1}(\cdot)$ and $s_{2}(\cdot)$, we have $\beta=\frac{\gamma_{1}^{\prime} \cdot \gamma_{3}}{\gamma_{2} \cdot \gamma_{5} \cdot \gamma_{4}}>1$. By Lemma 4 of Stampacchia [22], there exists a constant $C$ such that $\|u\|_{\infty} \leq C$.

Now let $p \in C_{+}(\bar{\Omega})$ be such that $p(x)<\frac{N p(x)}{N-p(x)}$ and $p(x)<(1+1 / N) p(x)$. By the continuity of $p(\cdot)$ on $\bar{\Omega}$ there exist constants $\delta_{1}, \delta_{2}>0$ such that

$$
\begin{array}{ll}
\max _{y \in B\left(x, \delta_{1}\right) \cap \Omega} p(y)<\min _{y \in \overline{B\left(x, \delta_{1}\right) \cap \Omega}} \frac{N p(y)}{N-p(y)} & \text { for all } x \in \bar{\Omega}, \\
\max _{y \in B\left(x, \delta_{2}\right) \cap \Omega} p(y)< & \text { inf }
\end{array}
$$

Since $\bar{\Omega}$ is compact, we can cover it with a finite number of balls $\left(B_{j}\right)_{j=1}^{k}$ and there exists a constant $\lambda>0$ such that

$$
\min \left(\delta_{1}, \delta_{2}\right)>\left|\Omega_{i}\right|>\lambda, \quad \Omega_{i}=B_{i} \cap \Omega, \quad \text { for } i=1, \ldots, k .
$$

We denote by $p_{j}^{+}$and $p_{* j}^{+}$the local maxima of $p$ and $p_{*}=\frac{N p}{N-p}$ on $\overline{\Omega_{j}}$ respectively (and by $p_{j}^{-}$and $p_{* j}^{-}$the respective local minima). By 2.9 and 
the fact that $p_{* i}^{-}<p_{*}=\frac{N p(\cdot)}{N-p(\cdot)}$ on $\Omega_{i}$, we have

$$
\int_{\Omega_{i}}\left|G_{k}(u)\right|^{p_{* i}^{-}} d x \leq c_{4}^{\prime} \max \left(\left(\Phi_{i}(k)\right)^{\frac{\left(\gamma_{1}^{i}\right)^{\prime} \cdot \gamma_{3}^{i}}{\gamma_{2}^{i} \cdot \gamma_{5}^{i} \cdot \gamma_{4}^{i}}} ;\left(\Phi_{i}(k)\right)^{\frac{\gamma_{3}^{i}}{\gamma_{6}^{i} \gamma_{4}^{i}}}\right) \quad \text { for } i=1, \ldots, k,
$$

with $\Phi_{i}(k)=\operatorname{meas}\left(\left\{x \in \Omega_{i}:|u|>k\right\}\right)$, and $\gamma_{j}^{i}$ the restriction of $\gamma_{j}$ to $\Omega_{i}$.

Choose $h$ such that $h-k>1$ and in $A_{h}^{i}=\left\{x \in \Omega_{i}:|u|>h\right\}$ we have $h-k<G_{k}(u)$. Then in view of 2.13) we obtain

$$
\Phi(h) \leq \frac{C}{(h-k)^{p_{* i}^{-}}} \max \left(\left(\Phi_{i}(k)\right)^{\frac{\left(\gamma_{1}^{i}\right)^{\prime} \cdot \gamma_{3}^{i}}{\gamma_{2}^{i} \cdot \gamma_{5}^{i} \cdot \gamma_{4}^{i}}} ;\left(\Phi_{i}(k)\right)^{\frac{\gamma_{3}^{i}}{\gamma_{6}^{2} \gamma_{4}^{i}}}\right) \quad \text { for } i=1, \ldots, k .
$$

It follows from 2.11 that

$$
\frac{\gamma_{3}^{j}}{\gamma_{4}^{j}}>1 \quad \text { and } \quad \frac{\left(\gamma_{1}^{j}\right)^{\prime}}{\gamma_{2}^{j}}>1 \quad \text { for all } x \in \bar{\Omega} \text { and } j=1, \ldots, k \text {, }
$$

which gives $\frac{\gamma_{3}^{j}}{\gamma_{4}^{j}} \frac{\left(\gamma_{1}^{j}\right)^{\prime}}{\gamma_{2}^{j}}>1$ and by a suitable choice of $s_{1}(\cdot)$ and $s_{2}(\cdot)$ we have

$$
\frac{\left(\gamma_{1}^{i}\right)^{\prime} \cdot \gamma_{3}^{i}}{\gamma_{2}^{i} \cdot \gamma_{5}^{i} \cdot \gamma_{4}^{i}}>1 \quad \text { for all } x \in \bar{\Omega} \text { and } i=1, \ldots, k \text {. }
$$

By [22, Lemma 4] we get $\|u\|_{\infty} \leq C$.

\section{Existence of solution for the unilateral problem (1.1)}

THEOREM 3.1. Suppose that assumptions $\left(H_{1}\right)-\left(H_{6}\right)$ hold, and let $q(x)>$ $\max \left(\frac{N}{p(x)}, p^{\prime}(x)\right)$ and $r(x)>\frac{N}{p(x)-1}$ for all $x \in \Omega$. Then there exists a weak solution $u$ to problem (1.1) (in the sense of definition (2.7)).

Proof. We divide the proof into three steps.

Step 1: A priori estimate. Let us define

$$
a_{n}(x, s, \xi)=a\left(x, T_{n}(s), \xi\right), \quad \text { a.e. } x \in \Omega, \forall s \in \mathbb{R}, \forall \xi \in \mathbb{R}^{N},
$$

and

$$
\begin{aligned}
\phi_{n}(s)=\phi\left(T_{n}(s)\right), \quad g_{n}(x, s)=T_{1 / n}(g(x, s)), \\
\text { a.e. } x \in \Omega, \forall s \in \mathbb{R}, \forall \xi \in \mathbb{R}^{N} .
\end{aligned}
$$

We consider the following approximate problem: find $u_{n} \in K(\psi)$ such that

$$
\begin{aligned}
& \left\langle-\operatorname{div}\left(a_{n}\left(x, u_{n}, \nabla u_{n}\right)\right), u_{n}-v\right\rangle+\left\langle-\operatorname{div}\left(\phi_{n}(u)\right), u_{n}-v\right\rangle \\
+ & \left(g_{n}\left(x, u_{n}\right), u_{n}-v\right) \leq\left(f, u_{n}-v\right)+\left\langle-\operatorname{div}(F), u_{n}-v\right\rangle \quad \forall v \in K(\psi) .
\end{aligned}
$$

By the classical result by Leray and Lions [16], for each $n \in \mathbb{N}$, there exists a weak solution $u_{n} \in K(\psi) \cap L^{\infty}(\Omega)$ of $(3.3)$. By the same argument as 
before, we derive that

$$
\left\|u_{n}\right\|_{L^{\infty}(\Omega)} \leq M
$$

and thus

$$
a_{n}\left(x, u_{n}, \nabla u_{n}\right)=a\left(x, u_{n}, \nabla u_{n}\right) \quad \text { and } \quad \phi_{n}\left(u_{n}\right)=\phi\left(u_{n}\right) .
$$

As $\psi \in K(\psi) \cap L^{\infty}(\Omega)$, taking $v=\psi$ as a test function in (3.3), by (3.5) we obtain

$$
\begin{array}{r}
\int_{\Omega} a\left(x, u_{n}, \nabla u_{n}\right) \nabla\left(u_{n}-\psi\right) d x+\int_{\Omega} \phi\left(u_{n}\right) \nabla\left(u_{n}-\psi\right) d x+\int_{\Omega} g\left(x, u_{n}\right)\left(u_{n}-\psi\right) d x \\
\leq \int_{\Omega} f\left(u_{n}-\psi\right) d x+\int_{\Omega} F \nabla\left(u_{n}-\psi\right) d x .
\end{array}
$$

Noting that $\int_{\Omega} \phi\left(u_{n}\right) \nabla u_{n} d x=0$, by Young's inequality, 3.5$)$ and $\left(H_{1}\right)-\left(H_{4}\right)$ we obtain

$$
\int_{\Omega}\left|\nabla u_{n}\right|^{p(x)} d x \leq C_{1} .
$$

By Proposition 2.2 and the last inequality,

$$
\left\|\nabla u_{n}\right\|_{L^{p(\cdot)}(\Omega)} \leq C_{2} .
$$

Then it follows from the results of [10] that there exists a subsequence of $\left(u_{n}\right)$ (still denoted by $\left(u_{n}\right)$ ) such that

$$
\begin{array}{cl}
\nabla u_{n} \rightarrow \nabla u & \text { weakly in }\left(L^{p(\cdot)}(\Omega)\right)^{N}, \\
u_{n} \rightarrow u & \text { strongly in } L^{p(\cdot)}(\Omega) \text { and a.e. in } \Omega, \\
u_{n} \rightarrow u & \text { weakly* in } L^{\infty}(\Omega) .
\end{array}
$$

By (3.8), we obtain

$$
g_{n}\left(x, u_{n}\right) \rightarrow g(x, u) \quad \text { a.e. in } \Omega .
$$

By assumption $\left(H_{4}\right)$ and (3.4), for any measurable set $E \subset \Omega$,

$$
\int_{E}\left|g_{n}\left(x, u_{n}\right)\right| d x \leq \int_{E} h_{M}(x) d x .
$$

Using Vitali's theorem, we conclude that

$$
g_{n}\left(x, u_{n}\right) \rightarrow g(x, u) \quad \text { strongly in } L^{1}(\Omega) .
$$

SteP 2: Almost everywhere convergence of the gradient. By $\left(H_{2}\right)$, to obtain the convergence of the gradient, it suffices to prove

$$
\limsup _{n \rightarrow \infty} \int_{\Omega}\left[a\left(x, u_{n}, \nabla u_{n}\right)-a\left(x, u_{n}, \nabla u\right)\right]\left(\nabla u_{n}-\nabla u\right) d x \leq 0 .
$$


The left-hand side of (3.11) can be written as

$$
\begin{aligned}
& \int_{\Omega}\left[a\left(x, u_{n}, \nabla u_{n}\right)-a(x, u, \nabla u)\right]\left(\nabla u_{n}-\nabla u\right) d x \\
& =\int_{\Omega} a\left(x, u_{n}, \nabla u_{n}\right)\left(\nabla u_{n}-\nabla u\right) d x-\int_{\Omega} a\left(x, u_{n}, \nabla u\right)\left(\nabla u_{n}-\nabla u\right) d x \\
& =A_{n}-B_{n} .
\end{aligned}
$$

The term $B_{n}$ goes to zero as $n \rightarrow \infty$. Indeed, by $\left(H_{2}\right)$, (3.4), (3.8), and Lebesgue's dominated convergence theorem, we have

$$
a\left(x, u_{n}, \nabla u\right) \rightarrow a(x, u, \nabla u) \quad \text { strongly in }\left(L^{p^{\prime}(\cdot)}(\Omega)\right)^{N} ;
$$

this convergence together with (3.7) implies $\lim _{n \rightarrow \infty} B_{n}=0$.

Next, we claim that $\limsup _{n \rightarrow \infty} A_{n} \leq 0$. Indeed, as $u_{n} \in K(\psi)$, and $u_{n} \rightarrow u$ almost everywhere, we deduce that $u \geq \psi$ a.e. in $\Omega$ and $u \in L^{\infty}(\Omega)$. Thus we can take $u$ as a test function in $(3.3)$. By (3.5), we obtain

$$
\begin{array}{r}
\int_{\Omega} a\left(x, u_{n}, \nabla u_{n}\right) \nabla\left(u_{n}-u\right) d x+\int_{\Omega} \phi\left(u_{n}\right) \nabla\left(u_{n}-u\right) d x+\int_{\Omega} g\left(x, u_{n}\right)\left(u_{n}-u\right) d x \\
\leq \int_{\Omega} f\left(u_{n}-u\right) d x+\int_{\Omega} F \nabla\left(u_{n}-u\right) d x .
\end{array}
$$

Letting $n \rightarrow \infty$ we get

$$
\limsup _{n \rightarrow \infty} A_{n} \leq 0 .
$$

By (3.13), and (3.14) and using Lemma 2.5 we conclude that

$$
\nabla u_{n} \rightarrow \nabla u \quad \text { a.e. in } \Omega \text {. }
$$

SteP 3: Passage to the limit. Let us take $v \in K(\psi) \cap L^{\infty}(\Omega)$ as a test function in $(3.3)$ :

$$
\begin{aligned}
\int_{\Omega} a\left(x, u_{n}, \nabla u_{n}\right) \nabla\left(u_{n}-v\right) d x & +\int_{\Omega} \phi\left(u_{n}\right) \nabla\left(u_{n}-v\right) d x \\
& +\int_{\Omega} g\left(x, u_{n}\right)\left(u_{n}-v\right) d x \\
\leq & \int_{\Omega} f\left(u_{n}-v\right) d x+\int_{\Omega} F \nabla\left(u_{n}-v\right) d x .
\end{aligned}
$$

By $\left(H_{3}\right)$ and the assumptions of the theorem it is easy to get

$$
\begin{aligned}
\int_{\Omega} f\left(u_{n}-v\right) d x & \rightarrow \int_{\Omega} f(u-v) d x, \\
\int_{\Omega} F \nabla\left(u_{n}-v\right) d x & \rightarrow \int_{\Omega} F \nabla(u-v) d x .
\end{aligned}
$$


Also by $\left(H_{4}\right),\left(H_{5}\right)$ and 3.4 ,

$$
\int_{\Omega} \phi\left(u_{n}\right) \nabla\left(u_{n}-v\right) d x \rightarrow \int_{\Omega} \phi(u) \nabla(u-v) d x
$$

and

$$
\int_{\Omega} g\left(x, u_{n}\right)\left(u_{n}-v\right) d x \rightarrow \int_{\Omega} g(x, u)(u-v) d x .
$$

For the first term in 3.16$)$, by 3.15 and $\left(H_{2}\right)$ we obtain

$$
a\left(x, u_{n}, \nabla u_{n}\right) \rightarrow a(x, u, \nabla u) \quad \text { strongly in }\left(L^{\left.p^{\prime} \cdot\right)}(\Omega)\right)^{N} .
$$

According to 3.7 , we have

$$
\int_{\Omega} a\left(x, u_{n}, \nabla u_{n}\right) \nabla\left(u_{n}-v\right) d x \rightarrow \int_{\Omega} a(x, u, \nabla u) \nabla(u-v) d x .
$$

Finally, by 3.17), 3.19, 3.22 we conclude that $u$ is a weak solution to problem (1.1).

4. Uniqueness of solution for (1.1). In this section, we discuss the uniqueness of weak solutions to problem (1.1). We make the following assumptions:

$\left(H_{6}\right) \phi$ is a locally Lipschitz continuous function.

$\left(H_{7}\right)$ For every $k>0$, there exists $\bar{c}_{k} \in L^{p^{\prime}(\cdot)}(\Omega)$ and a constant $\beta_{k}>0$ such that

$\left|a\left(x, s_{1}, \xi\right)-a\left(x, s_{2}, \xi\right)\right| \leq\left|s_{1}-s_{2}\right|\left[\beta_{k}|\xi|^{p(x)-1}+\bar{c}_{k}(x)\right] \quad$ for a.e. $x \in \Omega$ for all $\xi \in \mathbb{R}^{N}$ and $s_{1}, s_{2}$ with $\left|s_{1}\right|,\left|s_{2}\right| \leq k$.

$\left(H_{8}\right) g: \Omega \times \mathbb{R} \rightarrow \mathbb{R}$ is strictly increasing with respect to the second variable.

TheOREM 4.1. Suppose that $1<p(\cdot)<N$. Assume that $\left(H_{1}\right)-\left(H_{8}\right)$ hold. Then problem (1.1) admits a unique weak solution $u \in K(\psi) \cap L^{\infty}(\Omega)$.

Proof. The existence is proved in Theorem 3.1. Now, to prove uniqueness, assume that $u_{1}, u_{2} \in K(\psi) \cap L^{\infty}(\Omega)$ are two weak solutions to (1.1), SO

$$
\begin{aligned}
& \int_{\Omega} a\left(x, u_{1}, \nabla u_{1}\right) \nabla\left(u_{1}-v\right) d x+\int_{\Omega} \phi\left(u_{1}\right) \nabla\left(u_{1}-v\right) d x \\
& \quad+\int_{\Omega} g\left(x, u_{1}\right)\left(u_{1}-v\right) d x \\
& \leq \int_{\Omega} f\left(u_{1}-v\right) d x+\int_{\Omega} F \nabla\left(u_{1}-v\right) d x, \quad \forall v \in K(\psi) \cap L^{\infty}(\Omega),
\end{aligned}
$$


and

$$
\begin{aligned}
& \int_{\Omega} a\left(x, u_{2}, \nabla u_{2}\right) \nabla\left(u_{2}-v\right) d x+\int_{\Omega} \phi\left(u_{2}\right) \nabla\left(u_{2}-v\right) d x \\
& \quad+\int_{\Omega} g\left(x, u_{2}\right)\left(u_{2}-v\right) d x \\
& \leq \int_{\Omega} f\left(u_{2}-v\right) d x+\int_{\Omega} F \nabla\left(u_{2}-v\right) d x, \quad \forall v \in K(\psi) \cap L^{\infty}(\Omega) .
\end{aligned}
$$

Denote

$$
v_{1 \varepsilon}=u_{1}-T_{\varepsilon}\left(\left(u_{1}-u_{2}\right)^{+}\right), \quad v_{2 \varepsilon}=u_{2}+T_{\varepsilon}\left(\left(u_{1}-u_{2}\right)^{+}\right) .
$$

It is is easy to check that $v_{1 \varepsilon}, v_{2 \varepsilon} \in K(\psi) \cap L^{\infty}(\Omega)$. Thus, we can choose $v=v_{1 \varepsilon}$ and $v=v_{2 \varepsilon}$ as test functions in 4.2 and 4.3 to obtain

$$
\begin{aligned}
\frac{1}{\varepsilon} \int_{\Omega_{\varepsilon}}\left[a \left(x, u_{1}, \nabla\right.\right. & \left.\left.u_{1}\right)-a\left(x, u_{2}, \nabla u_{2}\right)\right] \nabla\left(u_{1}-u_{2}\right) d x \\
& +\frac{1}{\varepsilon} \int_{\Omega_{\varepsilon}}\left[\phi\left(u_{1}\right)-\phi\left(u_{2}\right)\right] \nabla\left(u_{1}-u_{2}\right) d x \\
& +\frac{1}{\varepsilon} \int_{\Omega_{\varepsilon}} T_{\varepsilon}\left(\left(u_{1}-u_{2}\right)^{+}\right)\left(g\left(x, u_{1}\right)-g\left(x, u_{2}\right)\right) d x \leq 0
\end{aligned}
$$

where $\Omega_{\varepsilon}=\left\{x \in \Omega: 0<u_{1}-u_{2}<\varepsilon\right\}$. Denote the three terms on the left-hand side by $J_{1}(\varepsilon), J_{2}(\varepsilon), J_{3}(\varepsilon)$. Then

$$
\begin{aligned}
J_{1}(\varepsilon) & =\frac{1}{\varepsilon} \int_{\Omega_{\varepsilon}}\left[a\left(x, u_{1}, \nabla u_{1}\right)-a\left(x, u_{1}, \nabla u_{2}\right)\right] \nabla\left(u_{1}-u_{2}\right) d x \\
& +\frac{1}{\varepsilon} \int_{\Omega_{\varepsilon}}\left[a\left(x, u_{1}, \nabla u_{2}\right)-a\left(x, u_{2}, \nabla u_{2}\right)\right] \nabla\left(u_{1}-u_{2}\right) d x .
\end{aligned}
$$

By Theorem 3.1, $\left\|u_{1}\right\|_{L^{\infty}(\Omega)},\left\|u_{2}\right\|_{L^{\infty}(\Omega)} \leq M$. Therefore, using $\left(H_{7}\right)$, we have

$$
\begin{aligned}
& \left|\frac{1}{\varepsilon} \int_{\Omega_{\varepsilon}}\left[a\left(x, u_{1}, \nabla u_{2}\right)-a\left(x, u_{2}, \nabla u_{2}\right)\right] \nabla\left(u_{1}-u_{2}\right) d x\right| \\
& \leq \int_{\Omega_{\varepsilon}}\left[\beta_{M}\left|\nabla u_{2}\right|^{p(x)-1}+c_{k}(x)\right] \nabla\left(u_{1}-u_{2}\right) d x .
\end{aligned}
$$

It follows that

$$
\lim _{\varepsilon \rightarrow \infty} \frac{1}{\varepsilon} \int_{\Omega_{\varepsilon}}\left[a\left(x, u_{1}, \nabla u_{2}\right)-a\left(x, u_{2}, \nabla u_{2}\right)\right] \nabla\left(u_{1}-u_{2}\right) d x=0 .
$$

Combining 4.6-4.7 with $\left(H_{2}\right)$ yields

$$
\limsup _{\varepsilon \rightarrow \infty} J_{1}(\varepsilon) \geq 0 \text {. }
$$


For the term $J_{2}$ we have, in view of $\left(H_{7}\right)$,

$$
\left|\frac{1}{\varepsilon} \int_{\Omega_{\varepsilon}}\left[\phi\left(u_{1}\right)-\phi\left(u_{2}\right)\right] \nabla\left(u_{1}-u_{2}\right) d x\right| \leq k_{M} \int_{\Omega_{\varepsilon}}\left|\nabla\left(u_{1}-u_{2}\right)\right| d x,
$$

where $k_{M}$ is the Lipschitz constant of $\phi$ on $[-M, M]$, and thus

$$
\lim _{\varepsilon \rightarrow \infty} J_{2}(\varepsilon)=0 .
$$

By $\left(H_{9}\right)$, it is easy to see that

$$
\begin{aligned}
\lim _{\varepsilon \rightarrow \infty} J_{3}(\varepsilon) & =\int_{\left\{u_{1} \geq u_{2}\right\}}\left(g\left(x, u_{1}\right)-g\left(x, u_{2}\right)\right) d x \\
& =\int_{\left\{u_{1}>u_{2}\right\}}\left(g\left(x, u_{1}\right)-g\left(x, u_{2}\right)\right) d x .
\end{aligned}
$$

Letting $\varepsilon \rightarrow \infty$, it follows from 4.8 4.10 that

$$
\int_{\left\{u_{1}>u_{2}\right\}}\left(g\left(x, u_{1}\right)-g\left(x, u_{2}\right)\right) d x \leq 0 .
$$

Hence, $\left|\left\{u_{1}>u_{2}\right\}\right|=0$, that is, $u_{1} \leq u_{2}$ a.e. in $\Omega$ and changing the roles of $u_{1}$ and $u_{2}$, we obtain $u_{2} \leq u_{1}$ a.e. in $\Omega$, which gives $u_{1}=u_{2}$ a.e. in $\Omega$.

\section{References}

[1] E. Acerbi and G. Mingione, Regularity results for stationary electro-rheological fluids, Arch. Ration. Mech. Anal. 164 (2002), 213-259.

[2] E. Acerbi, G. Mingione and G. A. Seregin, Regularity results for parabolic systems related to a class of non-Newtonian fluids, Ann. Inst. H. Poincaré Anal. Non Linéaire 21 (2004), 5-60.

[3] L. Aharouch and J. Bennouna, Existence and uniqueness of solutions of unilateral problems in Orlicz spaces, Nonlinear Anal. 72 (2010), 3553-3565.

[4] B. Aharrouch, M. Boukhrij and J. Bennouna, Existence of solutions for a class of degenerate elliptic equations in $P(x)$-Sobolev spaces, Topol. Methods Nonlinear Anal. 51 (2018), 389-411.

[5] S. Antontsev and J. F. Rodrigues, On stationary thermo-rheological viscous flows, Ann. Univ. Ferrara Sez. VII Sci. Mat. 52 (2006), 19-36.

[6] M. B. Benboubker, E. Azroul and A. Barbara, Quasilinear elliptic problems with nonstandard growth, Electron. J. Differential Equations 2011, art. 62, 16 pp.

[7] M. Bendahmane and P. Wittbold, Renormalized solutions for nonlinear elliptic equations with variable exponents and $L^{1}$ data, Nonlinear Anal. 70 (2009), 567-583.

[8] P. Bénilan, L. Boccardo, T. Gallouët, R. Gariepy, M. Pierre and J. L. Vázquez, An $L^{1}$-theory of existence and uniqueness of solutions of nonlinear elliptic equations, Ann. Scuola Norm. Sup. Pisa Cl. Sci. 22 (1995), 241-273.

[9] L. Boccardo, D. Giachetti, J. I. Diaz and F. Murat, Existence and regularity of renormalized solutions for some elliptic problems involving derivatives of nonlinear terms, J. Differential Equations 106 (1993), 215-237. 
[10] L. Boccardo, F. Murat and J. P. Puel, Existence of bounded solutions for nonlinear elliptic unilateral problems, Ann. Mat. Pura Appl. 152 (1988), 183-196.

[11] S. Challal, A. Lyaghfouri and J. F. Rodrigues, On the A-obstacle problem and Hausdorff measure of its free boundary, Ann. Mat. Pura Appl. 191 (2012), 113-165.

[12] S. Challal, A. Lyaghfouri, J. F. Rodrigues and R. Teymurazyan, On the regularity of the free boundary for quasilinear obstacle problems, Interfaces Free Bound. 16 (2014), 359-394.

[13] Y. Chen, S. Levine and M. Rao, Variable exponent, linear growth functionals in image restoration, SIAM J. Appl. Math. 66 (2006), 1383-1406.

[14] X. L. Fan and D. Zhao, On the generalised Orlicz-Sobolev space $W^{k, p(x)}(\Omega)$, J. Gansu Educ. College 12 (1998), 1-6.

[15] V. Ferone, M. R. Posteraro and J. M. Rakotoson, $L^{\infty}$-estimates for nonlinear elliptic problems with p-growth in the gradient, J. Inequal. Appl. 3 (1999), 109-125.

[16] J.-L. Lions, Quelques méthodes de résolution des problèmes aux limites non linéaires, Dunod and Gauthier-Villars, Paris, 1969.

[17] D. Marcon, J. F. Rodrigues and R. Teymurazyan, Homogenization of obstacle problems in Orlicz-Sobolev spaces, Portugal. Math. 75 (2018), 267-283.

[18] M. Rakotoson, Existence of bounded solutions of some degenerate quasilinear elliptic equations, Comm. Partial Differential Equations 12 (1987), 633-676.

[19] J. F. Rodrigues, M. Sanchón and J. M. Urbano, The obstacle problem for nonlinear elliptic equations with variable growth and $L^{1}$-data, Monatsh. Math. 154 (2008), 303322.

[20] J. F. Rodrigues and R. Teymurazyan, On the two obstacles problem in Orlicz-Sobolev spaces and applications, Complex Var. Elliptic Equations 56 (2011), 769-787.

[21] M. Růžička, Electrorheological Fluids: Modelling and Mathematical Theory, Lecture Notes in Math. 1748, Springer, Berlin, 2000.

[22] G. Stampacchia, Le problème de Dirichlet pour les équations elliptiques du second ordre à coefficients discontinus, Ann. Inst. Fourier (Grenoble) 15 (1965), 189-258.

[23] C. Yazough, E. Azroul and H. Redwane, Existence of solutions for some nonlinear elliptic unilateral problems with measure data, Electron. J. Qual. Theory Differential Equations 2013, art. 43, 21 pp.

[24] D. Zhao, W. J. Qiang, X. L. Fan, On generalized Orlicz spaces $L^{p(x)}(\Omega)$, J. Gansu Sci. 9 (1997), 1-7.

[25] V. Zhikov, Averaging of functionals of the calculus of variations and elasticity theory, Izv. Akad. Nauk SSSR Ser. Mat. 50 (1986), 675-710 (in Russian).

[26] W. Zou and F. Li, Existence of solutions for degenerate quasilinear elliptic equations, Nonlinear Anal. 73 (2010), 3069-3082.

[27] W. Zou, W. Wang and Y. Bi, On a class of nonlinear obstacle problems with nonstandard growth, Math. Methods Appl. Sci. 38 (2015), 2911-2921.

Benali Aharrouch, Jaouad Bennouna

Sidi Mohamed Ben Abdellah University

Faculty of Sciences Dhar El Mahraz

Laboratory LAMA, Department of Mathematics

P.O. Box 1796 Atlas

Fez, Morocco

E-mail: bnaliaharrouch@gmail.com

jbennouna@hotmail.com 
